\title{
Erratum
}

\section{Transdiagnostic and disorder-specific models of intergenerational transmission of internalizing pathology - ERRATUM}

\author{
L. R. Starr, C. C. Conway, C. L. Hammen and P. A. Brennan
}

doi:10.1017/S003329171300055X. Published online by Cambridge University Press, 24 April 2013.

The publisher apologizes for labelling errors introduced into Figure 1 of this article (Starr et al. 2013). The correct Figure 1 is given below.

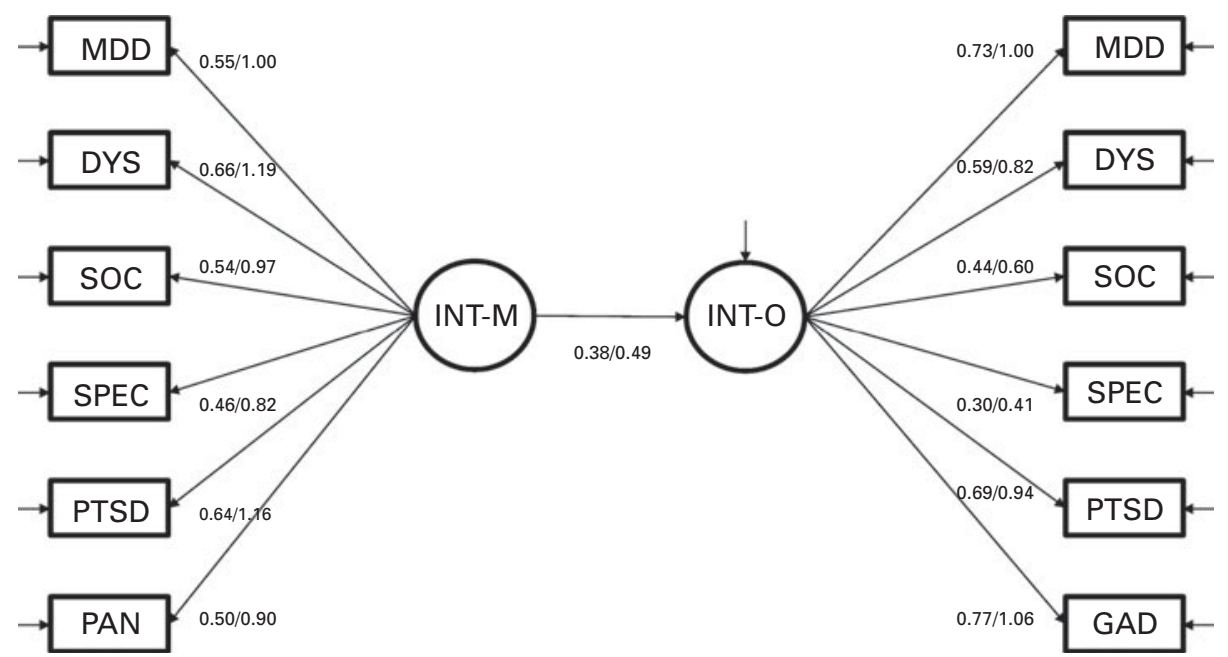

Fig. 1. A hierarchical-spectrum model of the intergenerational transmission of internalizing psychopathology. Coefficients are standardized/unstandardized. For clarity of presentation, the correlations between residual variances - representing intergenerational transmission of diagnosis-specific pathology - are not depicted (see Table 3). MDD, Major depressive disorder; DYS, dysthymia; SOC, social phobia; SPEC, specific phobia; PTSD, post-traumatic stress disorder; PAN, panic disorder or agoraphobia; INT-M, maternal internalizing factor (all observed variables loading on this latent factor reflect maternal diagnoses); INT-O, offspring internalizing factor (all observed variables loading on this factor reflect offspring diagnoses); GAD, generalized anxiety disorder.

\section{Reference}

Starr LR, Conway CC, Hammen CL and Brennan PA (2013). Transdiagnostic and disorder-specific models of intergenerational transmission of internalizing pathology. Psychological Medicine. doi:10.1017/ S003329171300055X. 